Prepared For SUbmission to JINST

International Conference "Instrumentation for Colliding Beam Physics"

FEBRUARY 24-28, 2020

Budker Institute of Nuclear Physics and Novosibirsk State University

\title{
Construction status of the Mu2e crystal calorimeter
}

\author{
a Joint Institute for Nuclear Research, Dubna, Russia \\ ${ }^{b}$ Laboratori Nazionali di Frascati dell'INFN, Frascati, Italy \\ ${ }^{c}$ INFN Sezione di Pisa, Pisa, Italy \\ ${ }^{d}$ California Institute of Technology, Pasadena, United States \\ e INFN Sezione di Lecce, Lecce, Italy \\ ${ }^{f}$ Università Guglielmo Marconi, Rome, Italy \\ ${ }^{g}$ Fermi National Laboratory, Batavia, Illinois, USA \\ ${ }^{h}$ Yale University, New Haven, USA \\ ${ }^{i}$ INFN Sezione di Trieste, Trieste, Italy \\ E-mail: rdonghia@lnf.infn.it
}

N. Atanov ${ }^{a}$ V. Baranov ${ }^{a}$ C. Bloise ${ }^{b}$ J. Budagov ${ }^{a}$ F. Cervelli $^{c}$ F. Colao ${ }^{b}$ M. Cordelli ${ }^{b}$

M. Corradi ${ }^{b}$ Yu.I. Davydov $^{a}$ S. Di Falco ${ }^{c}$ E. Diociaiuti ${ }^{b}$ S. Donati ${ }^{c}$ R. Donghia ${ }^{b 1}$ B. Echenard ${ }^{d}$

C. Ferrari ${ }^{c}$ S. Giovannella ${ }^{b}$ V. Glagolev $^{a}$ F. Grancagnolo ${ }^{e}$ D. Hampai ${ }^{b}$ F. Happacher ${ }^{b}$

D. Hitlin ${ }^{d}$ A. Marini ${ }^{c}$ M. Martini ${ }^{b, f}$ S. Miscetti ${ }^{b}$ T. Miyashita $^{d}$ L. Morescalchi ${ }^{c}$ P. Murat ${ }^{g}$

D. Pasciuto ${ }^{c}$ E. Pedreschi ${ }^{c}$ G. Pezzullo ${ }^{h}$ F. Porter $^{d}$ F. Raffaelli ${ }^{c}$ A. Saputi ${ }^{b}$ I. Sarra ${ }^{b}$

F. Spinella ${ }^{c}$ G.F. Tassielli ${ }^{e}$ V. Tereshchenko ${ }^{a}$ Z. Usubov ${ }^{a}$ I.I. Vasilyev ${ }^{a}$ A. Zanetti ${ }^{i}$ R.Y. Zhu ${ }^{d}$

${ }^{1}$ Corresponding author. 
Aвstract: The Mu2e experiment at Fermilab will search for the charged-lepton flavor violating neutrino-less conversion of a negative muon into an electron in the field of an aluminum nucleus. The Mu2e detector is composed of a tracker, an electromagnetic calorimeter and an external veto for cosmic rays. The calorimeter plays an important role in providing excellent particle identification capabilities and a fast online trigger filter, while aiding the track reconstruction capabilities. Calorimeter requirements are to provide a large acceptance for $100 \mathrm{MeV}$ electrons and reach: i) a time resolution better than $0.5 \mathrm{~ns}$; ii) an energy resolution better than $10 \%$; and iii) a position resolution of $1 \mathrm{~cm}$. The calorimeter consists of two disks, each one made of 674 pure CsI crystals. Each crystal is readout by two large area $2 \times 3$ arrays of UV-extended SiPMs of $6 \times 6 \mathrm{~mm}^{2}$ dimensions. A large scale prototype (Module-0) has been tested at an electron beam. We report here the tests done to finalize the calorimeter design, the results obtained with Module- 0 and the status of production. At this time, the performance characteristics of $85 \%$ of the crystals and all of the SiPMs have been measured. The calorimeter engineering drawings have been completed and the large mechanical components are under fabrication. Analog and digital electronics have been prototyped and tested with irradiation dose. Their serial production is being organized. The calorimeter assembly phase is planned for mid-2020.

KEYwords: Calorimeters, Radiation-hard detectors, Solid State detectors, Calibration

ArXiv ePrint: ToUpload 


\section{Contents}

1 The Mu2e detectors 1

2 Tests on Module-0 2

3 Production phase of crystals and SiPMs 4

4 Status of analog and digital electronics 5

5 Status of calorimeter mechanics 6

6 Conclusion $\quad 8$

\section{The Mu2e detectors}

The Mu2e experiment [1] at Fermilab will search for the charged-lepton flavor violating neutrinoless coherent conversion of a negative muon into a single electron in the field of an aluminum nucleus. The process produces a mono-energetic electron with an energy slightly below the muon mass $(104.967 \mathrm{MeV})$. If no events will be observed, Mu2e will set an upper limit on the ratio between the conversion rate and the muon capture rate, R, improving by four orders of magnitude the current limit [2]. On the other hand, an observation of Charge Lepton Flavour Violation (CLFV) events would be a clear signature of New Physics (NP) beyond the Standard Model up to mass scales of nearly $10^{4} \mathrm{TeV}$, far beyond the direct search reach at colliders, complementing and extending other CLFV searches on a wide range of NP scenarios [3].

The Mu2e experiment is composed by three superconducting solenoidal magnets: i) the Production Solenoid (PS); ii) the Transport Solenoid (TS); and iii) the Detector Solenoid (DS). An intense pulsed negative muon beam is produced by $8 \mathrm{GeV}$ protons hitting a tungsten target (PS) and about $40 \%$ of the muon beam is stopped, about $10^{10} \mu$ 's/sec, on an aluminum stopping target (DS) after traveling inside a very long, curved series of solenoids (TS). The strong negative gradient of the Production Solenoid, from 4 to $2.5 \mathrm{~T}$, confines soft pions and increases the yield through magnetic reflection. The S-shaped Transport Solenoid efficiently transfers low energy, negatively charged particles while allowing a large fraction of pions to decay into muons. The Detector Solenoid has a graded magnetic field from $2 \mathrm{~T}$ to $1 \mathrm{~T}$ in the upstream region of the stopping target, to increase acceptance for Conversion Electron (CE) events, while having an uniform $1 \mathrm{~T}$ field in the detector region. Downstream of the aluminum target, the detector is composed of a tracker and an electromagnetic calorimeter that operate inside the evacuated $\left(10^{-4}\right.$ Torr $)$ DS region in a high radiation environment. An external veto for cosmic rays (CR) surrounds the DS and half of the TS, to veto the CR background offline with $99.99 \%$ efficiency. A High Precision Germanium Detector and a $\mathrm{LaBr}$ crystal constitute the Stopping Target Monitor, placed $35 \mathrm{~m}$ downstream of the stopping 

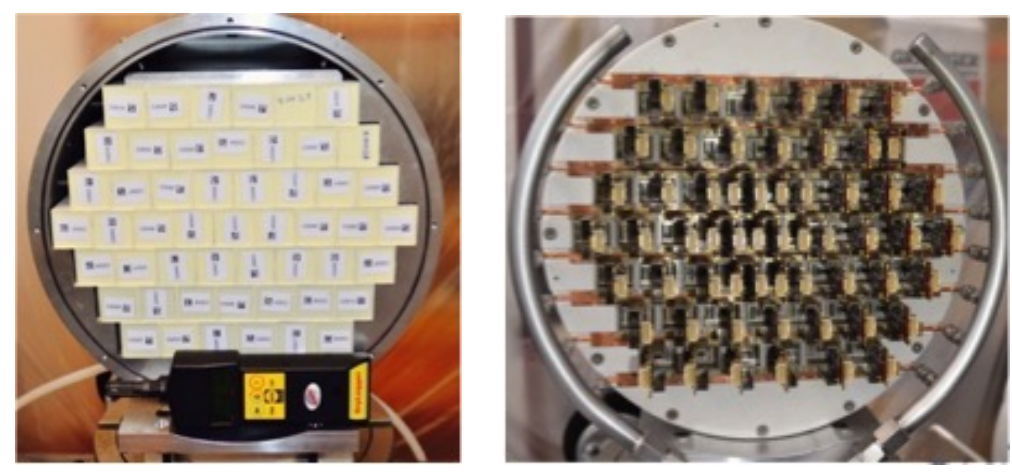

Figure 1. Pictures of the Module- 0 during assembly: front view with crystals (left) and back view with SiPM and FEE chips (right).

target, which provides normalization to CLFV events rate by detecting photons emitted from muon capture in the aluminum target.

The Mu2e tracker measures the CE momentum with a resolution $<180 \mathrm{keV}$ to separate the CE from the fast falling spectrum of the Decay in Orbit events. The calorimeter plays a complementary role to the tracking by providing excellent particle identification capabilities, a fast online trigger filter and a seed for track reconstruction. The calorimeter requirements are that of providing a large acceptance for the $\sim 100 \mathrm{MeV} \mathrm{CE}$ and reach at these energies: i) a time resolution better than $0.5 \mathrm{~ns}$; ii) an energy resolution better than $10 \%$; and iii) a position resolution of $1 \mathrm{~cm}$. Moreover, the calorimeter should withstand a total ionizing dose up to $100 \mathrm{krad}$ and a neutron fluence up to $10^{12} \mathrm{n} / \mathrm{cm}^{2}$.

After an intense R\&D phase [4], the calorimeter consists of 1348 un-doped CsI crystals organized in two separated annular disks. For redundancy, each crystal is readout by two large area UV-extended Silicon Photomultipliers (SiPMs). The Front End (FEE) amplification and HV regulator boards are connected to the SiPM pins while the digitization of the signals is carried out by custom boards (DIRAC) located in nearby crates. A radioactive source and a laser system will be used to equalize channels, set the energy scale and monitor the time dependence of response and resolution changes. In addition, a cooling system will cool down the SiPMs to below $0^{\circ} \mathrm{C}$, to reduce the leakage current related to the high neutron flux. Several tests have been performed on single components and on a large area calorimeter prototype (Module-0), to confirm that the chosen design satisfies the Mu2e requirements. In the following section, we describe the Module- 0 assembly procedure and the test results. In the other sections, the production status and the plan for assembly are summarized.

\section{Tests on Module-0}

A long $\mathrm{R} \& \mathrm{D}$ phase with small calorimeter prototypes demonstrated that the selected CsI+SiPM design well satisfies the detector requirements [5]. Many more dedicated tests were performed on a large scale prototype, dubbed Module-0 (Fig. 1), which was built using pre-production components for a total of 51 crystals, $102 \mathrm{SiPMs}$ and 102 FEE boards [6]. Module-0 is a structure of staggered 

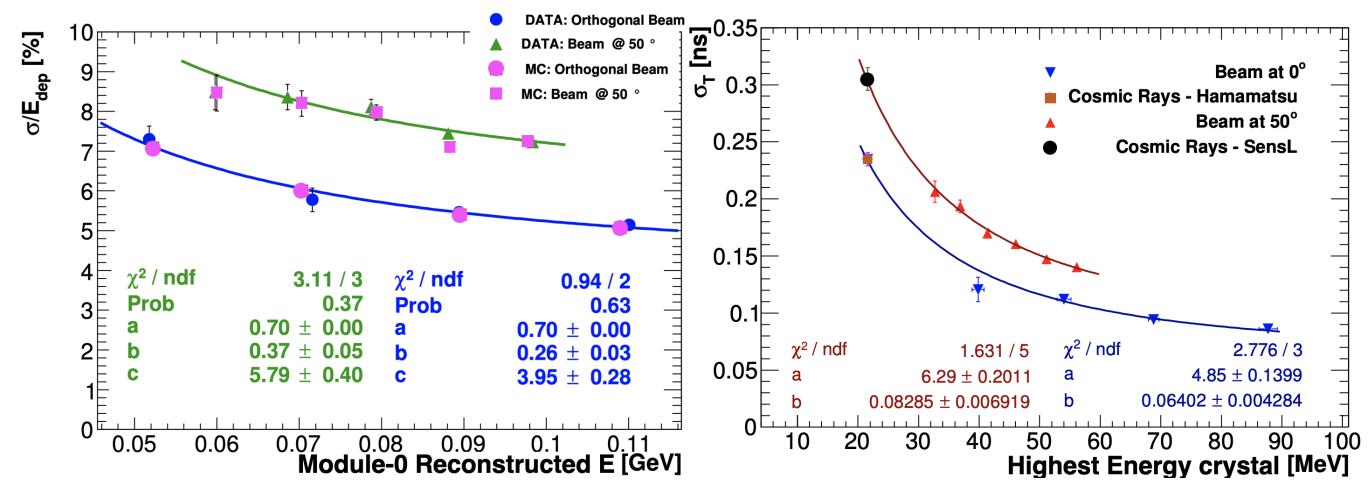

Figure 2. Left: Data-MC comparison of the energy resolution for all the test configuration. Right: Time resolution for all the test beam configuration; the results for CR data at $21 \mathrm{MeV}$ are also displayed.

crystals, representing a portion of the final disk structure, with a size large enough to contain most of the electromagnetic shower for $100 \mathrm{MeV}$ electrons. It has been used also to test integration and assembly procedures and to evaluate the operations of running in vacuum and at low temperatures. Module-0 performance was tested, at room temperature, with an electron beam in the energy range between 60 and $120 \mathrm{MeV}$ at the INFN Beam Test Facility in Frascati [7]. The energy and time resolutions were evaluated with particles impinging both at 0 and 50 degrees (expected CE incidence angle on the Mu2e calorimeter) on the calorimeter surface and also with $\mathrm{CR}$ data selected using a large plastic scintillator $\left(50 \times 50 \times 200 \mathrm{~mm}^{3}\right)$ located above the calorimeter. An energy resolution of $5.4 \%(7.5 \%)$ and a time resolution of $130 \mathrm{ps}(150 \mathrm{ps})$ are obtained for $100 \mathrm{MeV}$ particles impinging at 0 (50) degrees (Fig. 2). The results exceed the Mu2e physics requirements and a good Data-MC comparison was obtained.

This test beam concluded the R\&D phase and allowed us to start the production phase [8]. We are still using Module- 0 for continuing tests on calorimeter stability, and to study its operation in vacuum and with an upgraded version of the electronics. The "new" timing resolution obtained with CR data taking in vacuum is completely in agreement with the previous result reported in Fig. 2 (at $21 \mathrm{MeV}$ ). In addition to these tests, we performed a campaign of intense radiation hardness studies on pre-production samples, to evaluate the goodness of crystals and SiPMs [9] [10]. Crystals have shown to be dose and neutron resistant within requirements, while SiPMs suffer an increase of the leakage current, $I_{\text {dark }}$, as a function of the neutron fluence that makes them unusable when exceeding $2.5 \mathrm{~mA}$. In order to operate in the experiment, we need to cool them down to below $0^{\circ} \mathrm{C}$ and decrease the bias voltage, if necessary. In Fig. 3 (left) the dependence of leakage current for a sub-sample of irradiated production $\mathrm{SiPMs}$ is shown at $-10^{\circ} \mathrm{C}$ as a function of the bias voltage. In Fig. 3 (right), we show the distribution of timing resolution measured with laser pulses in Module-0 for different SiPM irradiation levels, when running at $0^{\circ} \mathrm{C}$ and as a function of the reconstructed charge. In this running condition, $\sim 1800 \mathrm{pC}$ correspond to $\sim 21 \mathrm{MeV}$ deposited energy of a MIP. This preliminary work demonstrated that the calorimeter performance can be met even at the end of the detector lifetime. Work is in progress to improve the algorithm used for timing reconstruction. 

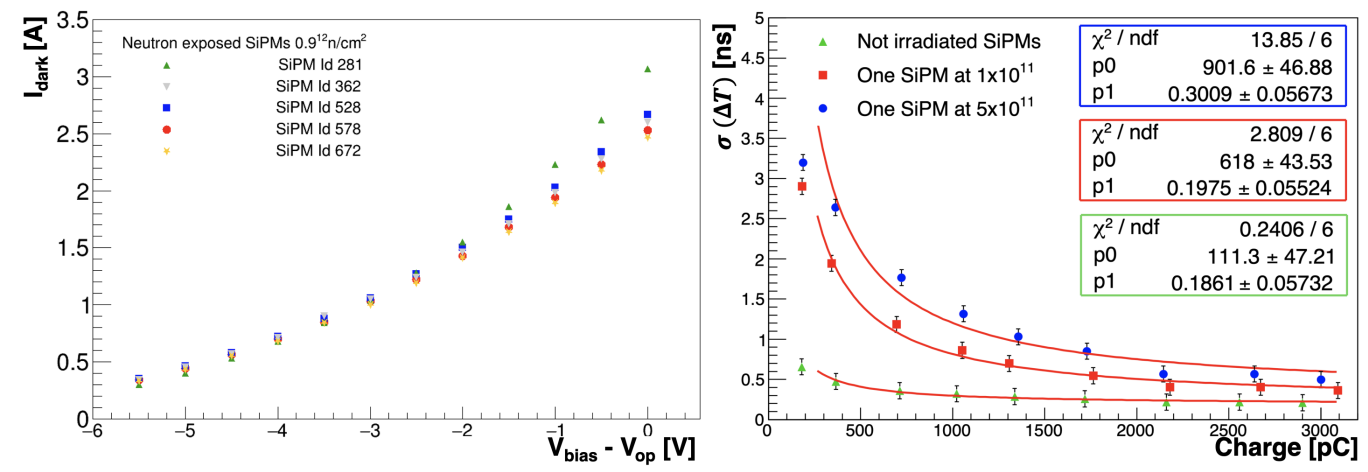

Figure 3. Left: Dark current of SiPMs irradiated up to $0.9 \times 10^{12} \mathrm{n} / \mathrm{cm}^{2}$ at $0^{\circ} \mathrm{C}$ as a function of the bias voltage . Right: Time resolution measured with laser pulses in Module-0 for a set of irradiated SiPMs, when operating at $0^{\circ} \mathrm{C}$, as a function of the reconstructed charge.

\section{Production phase of crystals and SiPMs}

The crystal and SiPMs production phase started in March 2018 [11]. At this time, $80 \%$ of the crystal production has been received and tested while the full production of 4,000 custom SiPMs have been successfully concluded. All crystals and sensors accepted exceed the required performance. Small samples, randomly selected along the production phase, have been irradiated with an ionization dose and neutron flux to characterize their radiation hardness.

Crystals were provided by two vendors: SICCAS (China) and Saint Gobain (SG, France). Crystals Quality Control starts with a dimensional control done with an Exagon Coordinate Measurement Machine, requiring a $0.1 \mathrm{~mm}$ tolerance with respect to nominal dimensions. Fig. 4 (left) shows the distribution of the crystal thickness for the measured crystals. Many SG crystals were rejected due to mechanical tolerances, thus causing a long delay in crystal procurement. For this reason, the remaining $20 \%$ of crystals production have been re-assigned to SICCAS. For crystals satisfying the dimensional requirement, the optical properties are measured using $511 \mathrm{keV}$ photons emitted by a ${ }^{22} \mathrm{Na}$ source [11]. In Fig. 4 (right) the number of photoelectrons per $\mathrm{MeV}$ is reported for both of the CsI producers. About $10 \%$ of the crystals have been rejected, mostly due to dimensional requirements. The irradiation tests carried out for the SiPMs and crystals subsamples demonstrated the calorimeter will be able to operate at the end of the Mu2e lifetime at a temperature below $0^{\circ} \mathrm{C}$.

For all of the 4,000 sensors, the breakdown voltage and the dark current have been measured at three different temperatures $\left(-10,0,25^{\circ} \mathrm{C}\right)$ [12]. The spread (RMS) of these quantities over the six SiPMs cells of the sensor array is used as a quality control parameter (Fig. 5). Due to a too large dark current RMS, the overall sensors rejection factor is lower than 1\%. Mean Time To Failure tests performed on randomly selected sensors, which maintained SiPM running for a few weeks at a warm-up temperature of $65^{\circ} \mathrm{C}$, allowed us to evaluate an MTTF value of 10 million hours, exceeding the experiment's needs by a factor of ten. 

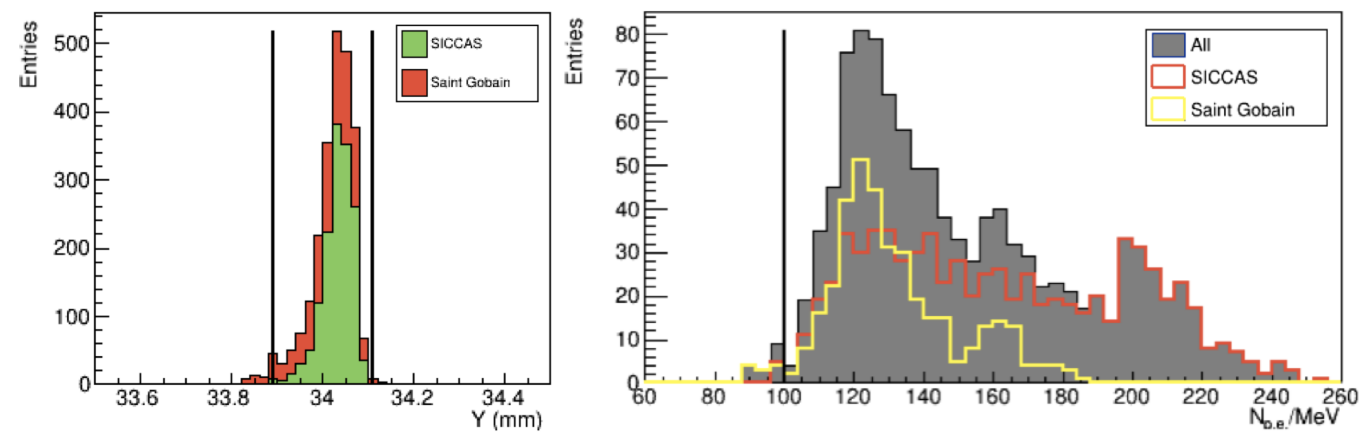

Figure 4. Left: Crystals thickness measured using a CMM machine. Right: Crystals light yield evaluated with $511 \mathrm{keV}$ electrons from a ${ }^{22} \mathrm{Na}$ source and a PMT readout.
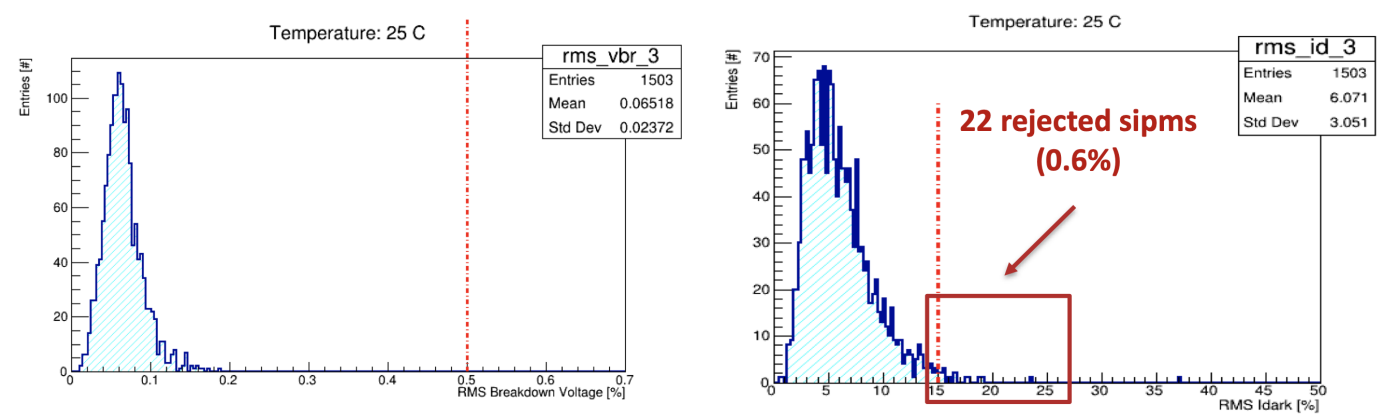

Figure 5. RMS of the SiPM breakdown voltage and dark current measured at $25^{\circ} \mathrm{C}$. Red lines represent the acceptance requirements.

\section{Status of analog and digital electronics}

In 2019 we completed the design of the FEE and the digitalization systems [13]. The FEE consists of two discrete and independent chips (Amp-HV) for each crystal, directly connected to the back of the SiPM pins. Each FEE board provides two amplification stages $(\times 1.5, \times 3)$, a local linear regulation of the bias voltage and monitoring of current and temperature on the sensors. The FEE is also needed to shape the sensor signal in order to have a $50 \mathrm{~ns}$ rise time and a full signal width of $250 \mathrm{~ns}$. The dynamic range is $2 \mathrm{~V}$. Groups of $20 \mathrm{Amp}-\mathrm{HV}$ chips are controlled by a dedicated mezzanine board (MB) equipped with an ARM processor to distribute the LV and the HV reference values and set and read back the locally regulated voltages. Groups of 20 signals are sent differentially to a digitizer module (DIRAC). The DIRAC board provides a further level of amplification and digitizes the SiPM signal at the sampling frequency of $200 \mathrm{MHz}$ with 12-bits ADC resolution. Digitized data are zero suppressed, merged, packed by an onboard FPGA and sent optically to the event builder using a custom protocol. The core of the board is the large FPGA that handles the ADCs protocol and timing, sparsifies and compresses the digitized data and forms a packet that is sent to the DAQ servers through optical fibers. In DIRAC v1 we used the MicroSemi SmartFusion2 MPF300 while in the V2 version we selected the MicroSemi PolarFire.

The expected total ionizing dose (TID) value for the digital electronics is of about $12 \mathrm{krad}$, while it is around $100 \mathrm{krad}$ in the FEE area. An intense irradiation campaign has been performed to accurately choose rad-hard electronic components, resistant to both TID and neutron and proton 
fluences.

Concerning the FEE design, in 2018 and 2019 we tested with neutrons and ionizing dose all the electronic components: voltage regulator; reference voltage sensor; temperature sensor; preamplifier; the ADC and DAC, which are used for reading and regulating high voltage and for monitoring current and temperature. A negligible deterioration was observed with neutron irradiation up to a total fluence of $10^{12} \mathrm{n} / \mathrm{cm}^{2}$. More than four radiation hardness tests with ionizing dose have been carried out to complete the FEE design and select rad-hard components. The most difficult components to qualify were the ADC and DAC. The selection of ADC128S022CIMT and DAC121S101CIMKX components from Texas Instrument [14] significantly improved the FEE functionality. In November 2019, the construction readiness review was carried out with satisfactory results. The final production phase has started in the early months of 2020 after having completed the last test on pre-production boards.

Also for the MB and DIRAC-v1 boards, rad-hard tests have been performed to check the functionality up the expected dose: total neutron fluence of $5 \times 10^{10} \mathrm{n} / \mathrm{cm}^{2}$ and $12 \mathrm{krad}$. Additional tests have been performed in a $1 \mathrm{~T}$ magnetic field and under vacuum. At this time, the second version of the DIRAC board has been designed and prototyped. The improvements were driven by radiation resistant requirements (changing FPGA, VTRX optical transceiver, DCDC converters). All the above mentioned tests have been repeated on DIRAC v2 boards, satisfying all requirements.

A complete slice test of the calorimeter was performed, to validate the analog part of the DIRAC and to check the entire readout electronic chain. This was done by connecting a certain number of crystals, SiPMs and FEE boards with voltage regulation given by the Mezzanine board and digital reading via the DIRAC (Fig. 6, left). The test was articulated into two steps to validate the system on physical signals: i) linearity test (DIRAC+MB+FEE+Charge injection; and ii) Cosmic ray readout of 16 Module- 0 channels. The resulting bandwidth and signal shape match simulation expectations. Tests provided results comparable to those achieved using a commercial CAEN digitizer, as shown in Fig. 6 (right).
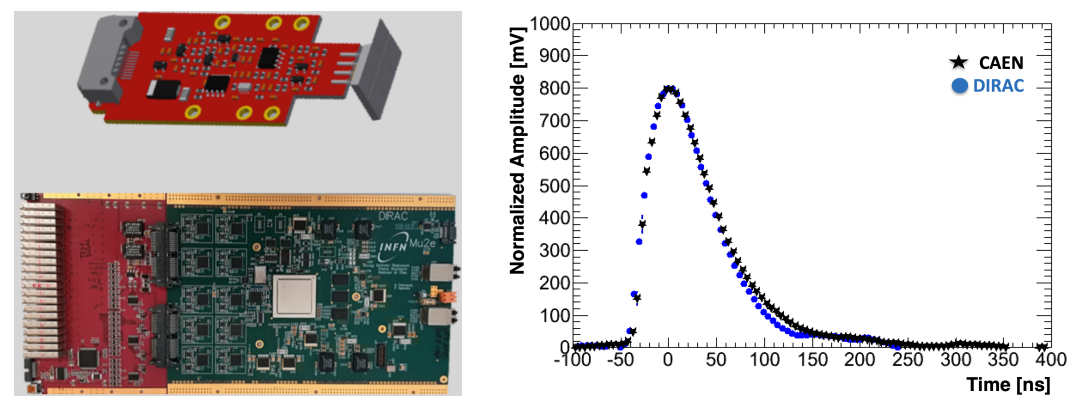

Figure 6. Left: CAD drawing of a FEE board connected to a Mu2e-SiPM (top) and picture of Mezzanine and DIRAC boards joined together. Right: CR signal obtained both with the final electronic readout chain and with a commercial CAEN digitizer.

\section{Status of calorimeter mechanics}

Each calorimeter annulus is composed of (Fig. 7): the outer Aluminum ring and the inner cylinders in composite material, the PEEK FEE plate, which is needed to insert, cool down and align the 
SiPMs to the corresponding crystals, the carbon fiber front face where the radioactive source pipes are integrated, and the MB+DIRAC crates mounted on the external cylinder (10 per disk). The Finite Element Analysis has been completed, showing a good stability of the system, with small stress on the feet.

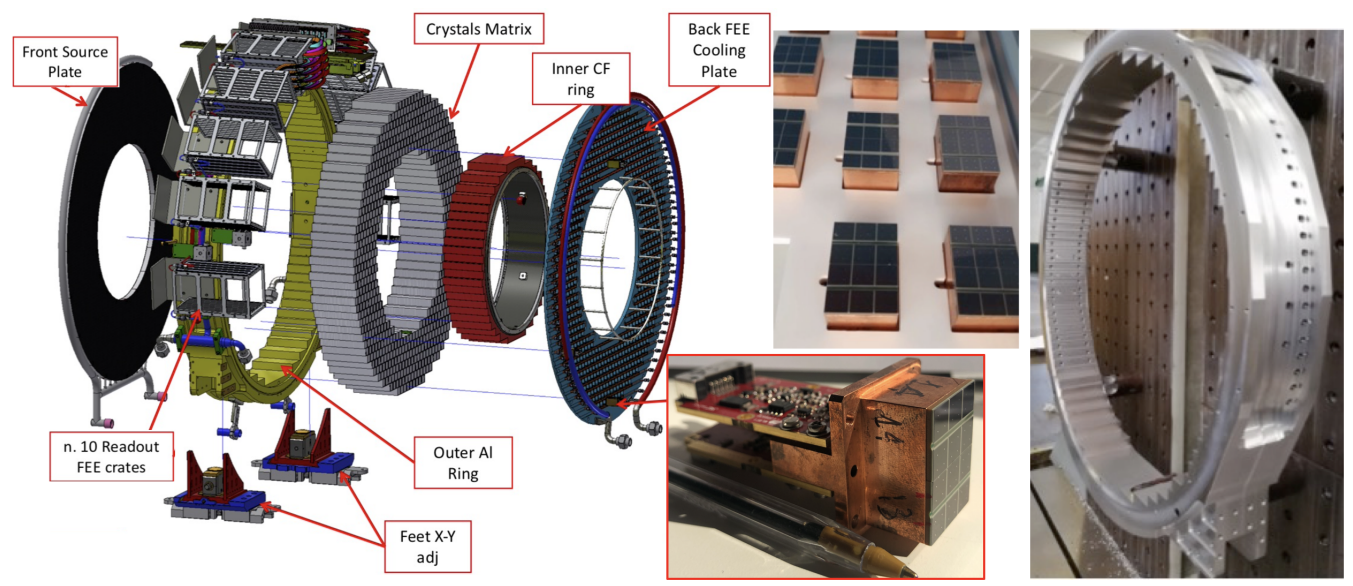

Figure 7. Left: exploded view of a calorimeter disk engineering model. Middle: few holders prototypes under glueing procedure. Right: first calorimeter outer Aluminum ring manufactured.

All materials used for the construction had to comply with outgassing properties required by the experiment and therefore we tested their behavior in vacuum. The inner stepped ring design was initially conceived to be a sandwich of carbon fiber skins and $\mathrm{Al}$ foam. The $\mathrm{Al}$ foam did not comply with the vacuum requirements also considering the issue that during the machining Aluminum debris could pollute the components. The latest design consider the use of Al honeycomb that is space compliant and the manufacturing and gluing of the parts will take place in a negative pressure environment to keep the parts clean.

A mockup with the final dimension has been developed and filled with iron fake crystals to study the assembly procedure and size all the construction tolerances. Crystals, wrapped with $150 \mu \mathrm{m}$ Tyvek and separated by a $20 \mu \mathrm{m}$ thick layer of black Tedlar, will be stacked from the bottom to the top inside the stepped external Aluminum cylindrical support. The alignment of each individual layer of crystals will be surveyed using a portable CMM. We have assessed the technique and procedure for the gluing of the SiPM to the copper holder. This, being in thermal contact with the cooling lines of the FEE plate, takes care of the thermalization of the SiPM and power dissipation of FEE boards. We have built the tooling set to precisely glue the SiPM to the holders and tested the procedure with a few prototype holders, as shown in Fig. 7 (middle). The laser calibration system layout is final. We have built the distribution chain for the laser light that illuminates each of the 674 crystals via quartz fibers and diffusive integrating spheres. At the end of 2019, all calorimeter structural parts are being produced. The calorimeter mechanical components have already been inserted in the GEANT4 simulation of the detector within the Mu2e software framework.

The two aluminum disks have been manufactured and are now being surveyed for QA (Fig. 7, right) along with all the copper SiPM/FEE holders. 


\section{Conclusion}

The Mu2e calorimeter is a state of the art crystal calorimeter with excellent energy $(<10 \%)$ and timing $(<500 \mathrm{ps})$ resolutions for $100 \mathrm{MeV} / \mathrm{c}$ electrons. A long R\&D phase demonstrated that pure $\mathrm{CsI}+\mathrm{SiPMs}$ design readout by a fast analog electronics and a digitization at $200 \mathrm{Msps}$ can satisfy Mu2e requirements and that this performance can be maintained also in vacuum and in presence of a radiation environment with a large ionization dose and neutron flux.

The calorimeter production phase started in March 2018 and is expected to be concluded within the end of 2020. The full SiPM production and $80 \%$ of the CsI crystals have been fully characterized. The electronic components have been fully qualified for functionality in B-field and in a high radiation environment. Electronics design both for analog and digital readout has been completed and production of the boards is under way. Production of the structural mechanical parts is under way and we plan to start the disk assembly in mid-2020. The calorimeter installation in the Mu2e experimental hall is planned for 2021 with a commissioning phase starting soon after.

\section{Acknowledgments}

We are grateful for the vital contributions of the Fermilab staff and the technical staff of the participating institutions. This work was supported by the US Department of Energy; the Istituto Nazionale di Fisica Nucleare, Italy; the Science and Technology Facilities Council, UK; the Ministry of Education and Science, Russian Federation; the National Science Foundation, USA; the Thousand Talents Plan, China; the Helmholtz Association, Germany; and the EU Horizon 2020 Research and Innovation Program under the Marie Sklodowska-Curie Grant Agreement No.690385 and No.734303. This document was prepared by members of the Mu2e Collaboration using the resources of the Fermi National Accelerator Laboratory (Fermilab), a U.S. Department of Energy, Office of Science, HEP User Facility. Fermilab is managed by Fermi Research Alliance, LLC (FRA), acting under Contract No. DE-AC02-07CH11359.

\section{References}

[1] L. Bartoszek et al., Mu2e Technical Design Report, arXiv:1501.05241, Fermilab-TM-2594, Fermilab-Design-2014-01.

[2] W.H. Bertl et al., SINDRUM II Collaboration, A search for $\mu-e$ conversion in muonic gold, Eur. Phys. J. C 47 (2006) 337.

[3] A. de Gouvea and P. Vogel, Lepton flavor and number conservation and physics beyond the standard model, Progress in Particle and Nuclear Physics 71 (2013) 75.

[4] N. Atanov et al., The Mu2e Calorimeter Final Technical Design Report, arXiv:1802.06341

[5] O. Atanova et al., Measurement of the energy and time resolution of a undoped CsI+MPPC array for the Mu2e experiment, JINST 12 (2017) P05007.

[6] A. Atanov et al., Design and test of the Mu2e undoped CsI + SiPM crystal calorimeter, NIM A 936 (2019) 94.

[7] G. Mazzitelli et al., Commissioning of the DAФNE beam test facility, Nucl. Instr. Meth. A 515 (2003). 
[8] N. Atanov, Electron beam test of the large area Mu2e calorimeter prototype, 2019 J. Phys.: Conf. Ser. 1162012027.

[9] M. Cordelli et al., Pre-production and quality assurance of the Mu2e calorimeter Silicon Photomultipliers, NIM A 912 (2018) 347.

[10] M. Cordelli et al., Neutron irradiation test of Hamamatsu, SensL and AdvanSiD UV-extended SiPMs, 2018 JINST 13 T03005.

[11] N. Atanov et al.,The Mu2e calorimeter: Quality assurance of production crystals and SiPMs, NIM A 936 (2019) 154.

[12] D. Caiulo et al., Production and Quality Assurance of the Mu2e Calorimeter Silicon Photomultipliers, J. Phys. Conf. Ser. 1162 (2019) 012024.

[13] N. Atanov et al., Mu2e Calorimeter Readout System, NIM A 936 (2019) 333.

[14] http://www.ti.com 